\title{
Palliativmedizin und Freitodbegleitung: Erfahrungsbericht einer Hausärztin
}

Erika Preisig

Korrespondenz: Dr. med. Erika Preisig Hausarztpraxis bim Brunne Langgartenstrasse 2 CH-4105 Biel-Benken

e.preisig@sunrise.ch
Biel-Benken, ein kleines, verschlafenes Dorf am äussersten Zipfel der Schweiz. Hier arbeite ich als überzeugte Hausärztin mit vielen Hausbesuchen und gut funktionierendem altherkömmlichem Notfalldienst und darauf bin ich stolz.

Ich habe in meiner 25-jährigen Praxiszeit viele Menschen sterben sehen. Ich habe immer versucht, meine Patienten zu Hause und nicht im Spital sterben zu lassen, wenn denn die letzten Tage kamen. Palliativmedizin lernte ich als eine «Disziplin» kennen, die im Studium stiefmütterlich behandelt worden war. Doch gerade an ihr fand ich Zufriedenheit, da ich dadurch meine Patienten zu Hause betreuen konnte bis zum letzten Moment.

2006 lernte ich dann, durch den Freitod meines Vaters, die wertvolle Arbeit von Exit und Dignitas kennen. Mein Vater schlief mit Natrium Pentobarbital (NAP) in meinen Armen ein, nachdem er einen Monat zuvor wegen schwerer Krankheit im Alter von 82 Jahren einen Tablettensuizidversuch bei mir zu Hause ausgeführt hatte. «Dank» seinem Entscheid lernte ich eine für mich fremde Art der Sterbehilfe kennen. Mein Vater ging mit einer unvorstellbaren Freude und Würde auf seinen letzten Weg.

Seither habe ich in 2 Fällen durch eine Freitodbegleitung einen Suizid durch Erschiessen verhindert, in beiden Fällen bei Patienten in Palliativsituaionen, die sich nicht pflegen lassen wollten. Ich habe wiederholt mit Exit und Dignitas zusammengearbeitet. An dieser Stelle möchte ich aber über meinen bewegendsten Fall berichten, bei dem ich mich dazu entschieden habe, ohne Unterstützung durch eine Organisation einen meiner Patienten in den Freitod zu begleiten. In diesem Zusammenhang möchte ich betonen, dass ich in meiner hausärztlichen Tätigkeit etwa alle 18 Monate eine Freitodbegleitung gutheisse, aber dreimal so oft einen Patienten palliativ in Zusammenarbeit mit der «Spitalexternen Onkologiepflege Baselland» (SEOP BL) und dem Hildegard Hospiz zu Hause begleite.

Herr W. war im Alter von 84 Jahren an einem Karzinom erkrankt. Er wurde wiederholt operiert, liess sich auf Radiotherapie und unzählige Chemotherapien ein. Bis zum Schluss glaubte er an eine Besserung, er lebte unglaublich gerne und war in einer sehr harmonischen Familie eingebettet. Herr W. lag mir sehr am Herzen. Er war eine angenehme, liebenswürdige Persönlichkeit, niemals beklagte er sich und war trotz seiner misslichen Lage immer zufrieden. Als der Onkologe ihm eröffnete, dass auch eine Chemotherapie nicht mehr sinnvoll sei und somit von seiner Seite her medizinisch wirklich nichts mehr für ihn getan wer- den könne, sprach ich mit ihm offen über die verschiedenen Formen des Sterbens. Ich klärte ihn über die Möglichkeiten der Palliativbegleitung zu Hause und auch über eine Freitodbegleitung auf, so wie ich das bei all meinen «austherapierten» Patienten tue. Eine Patientenverfügung hatte er schon lange unterschrieben.

Herrn W.s Gesundheitszustand verschlechterte sich zusehends. Als sich seine ihn pflegende Frau erschöpfte (auch schon über 80-jährig!), trat er ins Hildegard Hospiz ein, um dort «auf die Engelchen zu warten», wie er so schön sagte. Ich ging ihn einmal pro Woche besuchen; er beklagte sich nie und wartete geduldig auf das Ende. Nach 3 Wochen rief er mich an einem Sonntag an, ich müsse sofort kommen. Mein sonst so stolzer Herr W. sass am Fenster und war ein Hauch dessen, was er einst gewesen war. Er schaute in die Ferne und empfing mich mit den Worten: «Liebe Frau Doktor, Sie haben mir einmal etwas versprochen. Ich hoffe, dass Sie dieses Versprechen heute halten. Ich habe nun lange genug auf die Engelchen gewartet, doch die haben mich vergessen. Ich war ein Bankdirektor und habe mein ganzes Leben lang selber bestimmt, was für mich richtig ist. Ich werde mir niemals von einer Krankenschwester den Hintern putzen lassen. Nun schaffe ich es aber nicht mehr vom Bett auf den Toilettenstuhl. Dazuliegen und mich putzen zu lassen, ist für mich entwürdigend. Deswegen hoffe ich auf Ihre Hilfe. Ich gehe morgen nach Hause und Sie ermöglichen mir eine Freitodbegleitung, wie Sie mir dies versprochen haben.»

Zuerst glaubte ich, ihn falsch verstanden zu haben. Denn eine Freitodbegleitung innert zwei Tagen durchzuführen, ist mit beiden Organisationen völlig unmöglich. Dessen war ich mir bewusst. Doch die Gedankengänge und die Eigenwilligkeit meines lieben Patienten erinnerten mich an meinen Vater. Ich wollte alles tun, um mein damals gegebenes Versprechen einzuhalten. Ich gab Herrn W. den Auftrag, alles zu organisieren, damit er am nächsten Morgen um $11 \mathrm{Uhr}$ zu Hause sein könnte. Ich versprach ihm, meinerseits alles zu unternehmen, um die Freitodbegleitung auf $14 \mathrm{Uhr}$ zu realisieren. Nun begannen die Probleme. Exit oder Dignitas anzurufen, hatte in meinen Augen keinen Sinn. So rief ich diverse Apotheken in der Umgebung und schliesslich auch in Basel an, um das benötigte NAP zu erhalten. Fehlanzeige! NAP ist nur für die Veterinärmedizin zugelassen - da haben es die Tiere offenbar für einmal besser als wir. Kein Apotheker wollte einer Humanmedizinerin NAP abgeben. So rief ich eine Apotheke in Zürich an, von der ich wusste, dass sie für die Freitodorganisationen immer 
NAP auf Lager hat. Die Antwort war erlösend, der Apotheker wollte mir NAP gegen ein Rezept abgeben. Ein Verwandter von Herrn W. stellte sich zur Verfügung, nach Zürich zu fahren, um das Medikament zu holen. Erneut Fehlanzeige! NAP darf nur direkt an einen Arzt abgegeben werden, und eine Ausnahme machte der Apotheker nicht. Also fuhr ich persönlich nach Zürich.

Wie ich erwartet hatte, wurde es für Herrn W. nicht so einfach, aus dem Hospiz auszutreten und nach Hause zu gehen. Er war immer ein aufrichtiger Mensch gewesen, der zu seinen Entscheidungen stand. So hatte er der behandelnden Ärztin voller Freude erzählt, dass er entlassen werden wolle, um zu Hause in den Freitod gehen zu können. Diese mobilisierte alle und alles, um ihn davon abzuhalten. Es wurde ihm auch eine palliative Sedation angeboten, die er sofort ablehnte, da er sich ja nicht wie ein Säugling pflegen lassen wollte, auch nicht im komatösen Zustand. Er schaffte es, war um 12 Uhr zu Hause in seinem schönen Garten und erholte sich auf dem Liegestuhl. Dort wartete er voller Vertrauen auf seine Hausärztin, die ihm etwas versprochen hatte, das nicht ihrem Hippokratischen Eid, aber umso mehr ihrer humanen Einstellung entsprach.
Anstatt unangebrachte Fragen zu stellen, kondolierten sie der Witwe und involvierten das Institut für Rechtsmedizin. Die Staatsanwältin war erstaunt, dass keine Sterbehilfeorganisation vor Ort war, kondolierte nach einer sehr kurzen Untersuchung des Verstorbenen ebenfalls und liess mich nach Beschlagnahmung meiner Videoaufzeichnung die Todesbescheinigung ausstellen (zu meinem eigenen Schutz dokumentiere ich das Öffnen des Ventiles immer mit einer Videoaufzeichnung).

Es ist mir ein Anliegen, dass wir Hausärzte lernen, auch unangenehme Wünsche unserer Patienten zu akzeptieren. Hätte mich mein Vater nicht durch die Tatsache, dass er sich unter den Zug werfen wollte, dazu aufgefordert, ihm eine menschliche Art der Lebensbeendigung zu ermöglichen, hätte ich mich niemals mit Freitodbegleitung auseinandergesetzt. 5 Jahre nach seinem Tod bin ich ihm dankbar für diese «Weiterbildung».

Ich habe in einem Workshop in Arosa von Kollegen gehört, wie sie schwerstleidenden Patienten auf illegale Weise helfen, in den Tod zu gehen. Ich war entsetzt, denn wir haben in der Schweiz legale Möglichkeiten. Wir können unseren unheilbar kranken

\section{Es erstaunt mich bei jeder Freitodbegleitung immer wieder, wie zutiefst glücklich und dankbar der Betroffene ist. Im Gegensatz dazu, wie traurig, aber verständnisvoll und tapfer die Angehörigen sind.}

Es erstaunt mich bei jeder Freitodbegleitung immer wieder, wie zutiefst glücklich und dankbar der Betroffene ist. Im Gegensatz dazu, wie traurig, aber verständnisvoll und tapfer die Angehörigen sind. Wir wollten alle Herrn W. noch nicht gehen lassen, versuchten ihn zu überreden, noch wenigstens 2-3 Tage das schöne Sommerwetter $\mathrm{zu}$ Hause zu geniessen. Aber nur er selber konnte beurteilen, wie sehr er litt. Die Schmerzen, die nur mit einer Sedation hätten erträglich gemacht werden können, und die Erschöpfung, die dazu geführt hatte, dass er nicht mehr aufstehen konnte, waren für ihn ein nicht akzeptables Leiden. Schliesslich mussten wir aufgeben und begnügten uns damit, ihn im Sonnenschein seines geliebten Gartens noch einmal von seinem bewegten Leben erzählen zu lassen.

Um 15 Uhr öffnete Herr W. mit zittrigen, schwachen Händen das Ventil der Infusion. Seine Frau und seine Tochter sassen am Bett. Mit Tränen in den Augen hörten wir, wie er glücklich seine letzten Worte rief, «Sali zäme, jetzt gang I in Himmel!» Dann schlief er für immer ein, ohne ein Stöhnen, stolz wie mein Vater, und - im übertragenen Sinn - wie Reinhard May es singt, «Ich will im Stehen sterben ...»

Ich meldete der Polizei einen aussergewöhnlichen Todesfall, und zehn Minuten später klopften zwei überaus freundliche Polizisten an der Türe. und schwerstleidenden Patienten nicht nur die Palliativsedation, sondern auch die Freitodbegleitung bei vollem Bewusstsein und in Anwesenheit ihrer Liebsten anbieten. Warum haben so viele Ärzte Angst davor? Unsere Patienten werden nicht nur immer älter, sondern auch selbstbewusster und möchten auch ihr Lebensende selber bestimmen. Dies hat das Abstimmungsergebnis im Kanton Zürich deutlich bewiesen. (84 zu 16\% für eine Selbstbestimmung am Lebensende, sprich Möglichkeit einer Freitodbegleitung.)

Wenn ich wüsste, dass Interesse bei der Ärzteschaft vorhanden ist, würde ich sehr gerne eine Fortbildungsveranstaltung organisieren zu folgenden Fragen:

- Palliativmedizin und Freitodbegleitung: Schliesst das Eine das Andere aus?

- Was sind die Voraussetzungen für eine Freitodbegleitung durch den Hausarzt? Brauchen wir eine Freitodbegleitungsorganisation?

Die Begleitung meines Patienten Herrn W., meine erste Freitodbegleitung ohne eine Organisation, war ein überaus positives Erlebnis für mich. Ich hoffe für alle Schwerstleidenden, die vor sich selber und vor ihrem Schöpfer einen begleiteten Suizid verantworten können, dass es bald mehr Ärzte gibt, die den Wunsch nach einem begleiteten Abschied in Würde respektieren. 